\title{
Requiem pour l'ISH ou la fin de l'approche pluridisciplinaire inter-institutionnelle au Cameroun
}

François Tchala Abina

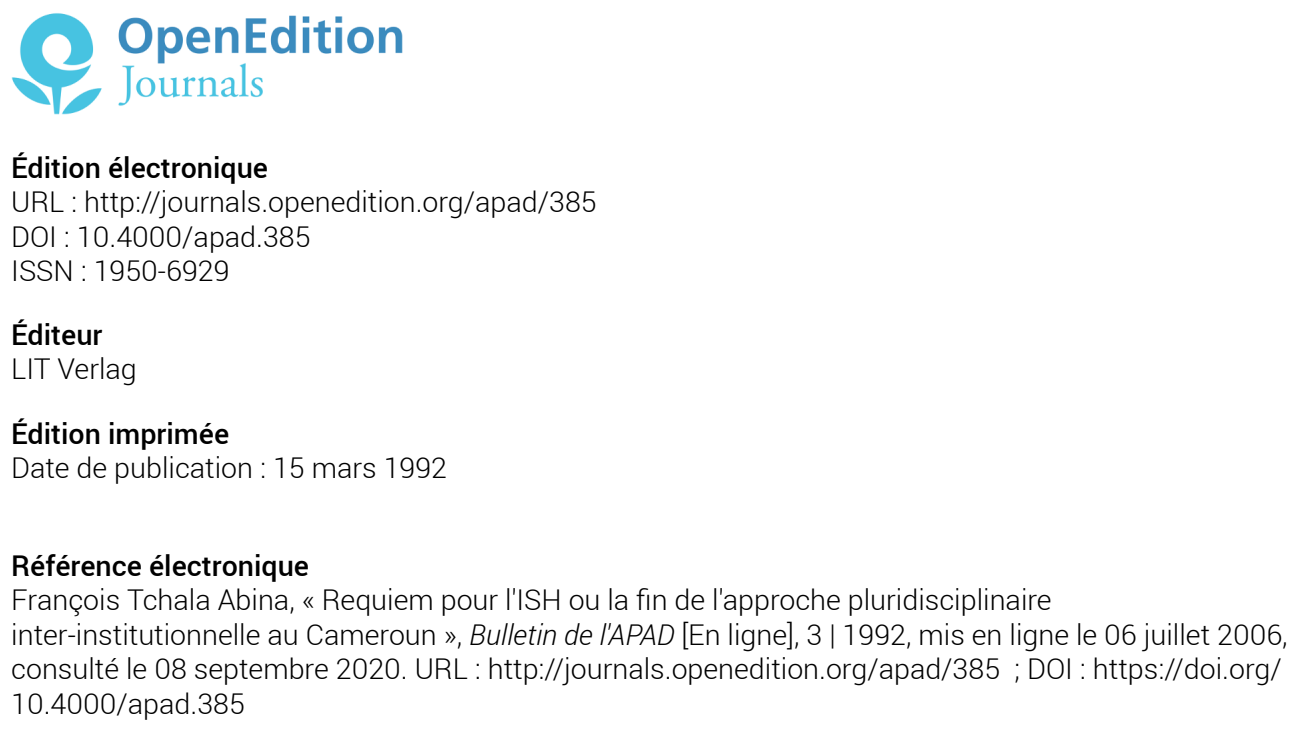

Ce document a été généré automatiquement le 8 septembre 2020

Bulletin de I'APAD 


\title{
Requiem pour l'ISH ou la fin de l'approche pluridisciplinaire inter-institutionnelle au Cameroun
}

\author{
François Tchala Abina
}

(Reproduit du Bulletin $n^{\circ} 5$ du Réseau OCISCA, avec l'autorisation de l'auteur)

2 Que le développement agricole soit multidimensionnel et de ce fait nécessite une approche pluridisciplinaire est désormais reconnu par tous: décideurs politiques, théoriciens et praticiens du développement. En clair, l'époque où la mise au point d'une variété à haut rendement ou d'une race extrêmement performante était considérée comme suffisante pour promouvoir le développement agricole d'une région est bel et bien révolue. Cette évolution s'est faite progressivement au prix de nombreux échecs, les approches sectorielles et thématiques ayant le plus souvent privilégié les aspects techniques au détriment des aspects socio-économiques et culturels. Ces derniers aspects s'étant, au fil des années, révélés d'importants facteurs limitants du développement agricole, leur prise en considération s'avérait indispensable. C'est ce qui explique l'implication croissante actuelle des spécialistes en sciences sociales dans l'élaboration et l'analyse des projets de développement et dans la mise au point des "paquets" technologiques. Au niveau de la recherche agricole, on a aussi assisté à la création de structures de recherche en sciences sociales appelées à collaborer avec les chercheurs en productions végétales et animales.

La récente décision du Gouvernement Camerounais de mettre fin à l'existence de l'Institut des Sciences Humaines (ISH) apparaît dans ce contexte comme allant à contre-courant de l'évolution actuelle de la conceptualisation de la problématique du développement agricole. Cette affirmation semble d'autant plus justifiée que la dissolution de l'ISH intervient au moment où l'Institut de Recherche Agronomique (IRA) et l'Institut de Recherche Zootechnique (IRZ) sont restructurés et appelés à être plus performants que par le passé.

4 En effet, la création de l'ISH avait été saluée en son temps par les spécialistes des problèmes de développement comme reflétant la prise de conscience par les autorités 
camerounaises de l'importance des facteurs socio-économiques et culturels en matière de développement. Bien qu'autonome, l'ISH était, entre autres missions, chargé d'apporter aux autres Instituts techniques et particulièrement à l'IRA et l'IRZ, les savoirs et savoir-faire en sciences humaines qui faciliteraient le transfert des paquets technologiques aux populations rurales cibles. En informant ces instituts des réalités économiques, démographiques et socio-culturelles du milieu rural camerounais, et des mutations intervenant au sein de celui-ci, les chercheurs en sciences humaines devaient permettre la mise au point et le transfert de technologies qui soient non seulement techniquement efficaces, mais aussi économiquement rentables et acceptables sur le plan socio-culturel. Cette triple exigence technique, économique et socio-culturelle impliquait une collaboration étroite en l'ISH et chacun des instituts techniques, en bref, une approche pluri-disciplinaire. Qu'en a-t-il été en réalité ?

Disons-le d'emblée et sans précaution oratoire inutile, le bilan de cette approche est globalement négatif. Sans méchanceté aucune, il est possible d'affirmer que l'enterrement de l'ISH se fait dans l'indifférence totale de l'IRA et de l'IRZ, qui n'ont pratiquement jamais bénéficié du soutien qu'ils étaient en droit d'attendre de l'ISH. "Les morts ayant toujours tort", il ne serait pas surprenant que l'absence de soutien de l'ISH aux Instituts techniques soit avancée comme une raison de plus justifiant sa disparition. Si le constat est évident, il n'en est pas de même de la culpabilité de l'ISH. L'approche pluri-disciplinaire qui aurait rendu l'ISH plus utile au développement du secteur agricole est extrêmement complexe. Elle s'accommode difficilement de la formation thématique reçue aussi bien par les chercheurs de l'ISB que par ceux des autres instituts. Elle est bloquée dans un contexte où les règles de gestion des ressources humaines et financières sont extrêmement rigides. Elle est inopérante dans le cadre de projets montés à la hâte en vue de l'obtention de financements conditionnés par une certaine pluridisciplinarité. En clair, elle est difficile à mettre en œuvre sans une reconnaissance effective, par les chercheurs, d'une certaine complémentarité de leurs disciplines et en l'absence d'une volonté politique réelle de la promouvoir. A partir du moment où, avec l'assistance de certains bailleurs de fonds, il a été permis à l'IRA et à l'IRZ de recruter directement leurs propres spécialistes en agro-économie, sociologie rurale et vulgarisation agricole, il n'était point besoin d'être devin pour prédire la disparition de l'ISB ou, tout au moins, celle de certains de ses organes vitaux... ce qui revient au même. C'est dire que le requiem d'aujourd'hui aurait tout aussi bien pu être chanté dix années auparavant

6 Comment, en cet instant, ne pas se rappeler avec une certaine nostalgie l'époque lointaine où N'Sangou Arouna, agro-économiste de l'ISB, se préoccupait de la rentabilité des engrais dans les essais de la recherche agronomique dans certaines régions du Cameroun?

7 La fermeture de l'ISB soulagera certainement l'État d'une charge. En cette période d'ajustement structurel, le social, y compris dans le domaine scientifique, subit souvent les premières coupes sombres. Il est toutefois possible d'espérer que le transfert de certains spécialistes de l'ISB à l'IRA et peut-être aussi à l'IRZ pourra accroître les chances de réussite de l'approche pluri-disciplinaire au sein de chacun de ces instituts, à la faveur de la suppression des anciennes barrières institutionnelles. Si cela advenait, alors la disparition de l'ISB aura été salutaire au moins sur ce plan. Nous ne saurions cependant nous départir d'un certain scepticisme, car l'approche pluridisciplinaire a 
des exigences qui vont au delà de la seule proximité physique des différents spécialistes.

\section{AUTEUR}

FRANÇOIS TCHALA ABINA

Agro-sociologue, INADER/CUDS 